\title{
16th International IUPAC Conference on High Temperature Materials Chemistry (Ekaterinburg, July 2-6, 2018)
}

16th International IUPAC Conference on High Temperature Materials Chemistry will be held July 2-6, 2018 in Ekaterinburg, Russian Federation.

\begin{tabular}{ll}
\multicolumn{2}{c}{ Organizers } \\
IUPAC & Ural Federal University (UrFU) \\
Govergment of Sverdlovsk Region & Ural Branch of the Russian Academy of Siences (UB RAS) \\
Ural State Pedagogical University (USPU) & Institute of Metallurgy (IMET), UB RAS
\end{tabular}

National Committee

\begin{abstract}
Chairman Academician N. A. Vatolin, Institute of Metallurgy, Ural Branch of RAS (Ekaterinburg)
Vice-chairman Academician N. V. Mushnikov, Ural Branch of RAS (Ekaterinburg)
\end{abstract}

\begin{abstract}
M. I. Alymov (Chernogolovka)
Yu. I. Biktuganov (Ekaterinburg)

V. V. Brazhkin (Troitsk)

V. N. Charushin (Ekaterinburg)

O. N. Chupakhin (Ekaterinburg)

K. S. Gavrichev (Moscow)

V. V. Gusarov (St. Petersburg)

B. M. Igoshev (Ekaterinburg)
\end{abstract}

Masaki Azuma (Japan)

Giovanni Balducci (Italy)

Catherine Bessada (France)

Ricardo Castro (USA)

Patrick Echegut (France)

Yingwei Fei (USA)

Nina Ilinykh (Russia)

\author{
E. N. Kablov (Moscow) \\ V. A. Koksharov (Ekaterinburg) \\ L. I. Leontiev (Moscow) \\ V. L. Lisin (Ekaterinburg) \\ A. I. Nikolaev (Apatity) \\ S. V. Perestoronin (Ekaterinburg) \\ A. A. Rempel (Ekaterinburg) \\ V. N. Rychkov (Ekaterinburg)
}

\section{International Advisory Board}

Herbert Ipser (Austria)

Joonho Lee (Korea)

Luis Filipe Malheiros (Portugal)

Tetsuo Mohri (Japan)

Alexandra Navrotsky (USA)

Shizhang Qiao (Australia)
V. Ya. Shevchenko (St. Petersburg)

A. A. Simonova (Ekaterinburg)

K. A. Solntsev (Moscow)

V. L. Stolyarova (St. Petersburg)

N. P. Tarasova (Moscow)

A. Yu. Tsivadze (Moscow)

H. P. Vyatkin (Chelyabinsk)

Gerd M. Rosenblatt (USA)

Valeriy Sidorov (Russia)

Hans Seifert (Germany)

Sharon Webb (Germany)

Ping Wu (Singapore)

Xianran Xing (China)

\begin{tabular}{ll}
\multicolumn{2}{c}{ Local Committee } \\
Conference Chair & Prof. Valeriy Sidorov (USPU) \\
Vice-Chairs & Dr. Nikolay Dubinin (IMET UB RAS), \\
& Dr. Ilya Polovov (UrFU) \\
Scientific secretary & Dr. Nina Ilinykh (UrTISI SIBSUTIS)
\end{tabular}

Main Topics

High temperature thermodynamic measurements

Theory and modeling of high temperature materials

Melts, ceramics, glasses and amorphous materials

Transport, ionic and electronic conductivity, grain boundaries, interfaces and surfaces

Phase structure and metallurgical processes, corrosion

Earth and planetary materials at high pressures and temperatures

Materials for nuclear energy applications

Materials for aerospace applications

Materials for advanced sources of energy

\section{Confirmed plenary speakers}
I. A. Abrikosov (Sweden)
Dirk Holland-Moritz ( Germany)
J. Irvine (United Kingdom)
V. Pralong (France)
A. Sundaresan (India)
X. Xianran (China)

Contacts: htmc16@gmail.com; htmc16@mail.ru

\section{www.htmc16.ru}

